\title{
STRUCTURE ET RÉGÉNÉRATION DES PEUPLEMENTS NATURELS DE BALANITES AEGYPTIACA (L.) DEL. ET ZIZIPHUS MAURITIANA LAM. SUIVANT UN GRADIENT ÉCOLOGIQUE DANS LA RÉGION DE MARADI AU NIGER
}

Rabiou Habou ${ }^{1}$, Moussa Massaoudou², Tougiani Abasse², Mahamane Ali ${ }^{3}$, Mahamane Larwanou ${ }^{4}$, Patrick Van Damme

1 Département des production végétales, Université de Diffa, Niger

2 Institut National de la Recherche Agronomique du Niger, Niger

3 Département de Biologie, Université Abdou Moumouni de Niamey, Niger

4 Faculté d'Agronomie, Université Abdou Moumouni de Niamey, Niger

5 Université de Gand, Belgique, et Czech University of Life Sciences, Prague,

Czech Republic

Les espèces ligneuses jouent un rôle important dans la vie des populations locales. Parmi les espèces couramment utilisées au Niger figurent Balanites aegyptiaca et Ziziphus mauritiana. Ces deux espèces sont exploitées comme sources alimentaires et pour divers produits médicinaux, avec comme conséquence une réduction de leurs peuplements. La présente étude vise à analyser la structure des peuplements et les modes de régénération des deux espèces dans deux secteurs agro-écologiques contrastées de la région de Maradi au centre sud du Niger. Au total, 60 relevés ont été délimités, d'une part dans des formations naturelles dominées par ces espèces, et d'autre part dans les systèmes agrosylvopastoraux des secteurs sahélien et sahélo-soudanien. Dans chaque placette et sur chaque arbre, le diamètre à 1,30 m au rasdu sol, la hauteur totale et deux diamètres perpendiculaires de houppier ont été mesurés. L'inventaire de la régénération a été effectué dans 5 placeaux. La nature de la régénération (drageon, marcotte, semis naturel), la hauteur totale et le nombre de tiges de chaque plantule ont été notés. La densité de $B$. aegyptiaca ne varie significativement entre sites et entre secteurs. Par contre, la densité de $Z$. mauritiana varie significativement entre sites et entre secteurs. En effet, le site de Birni Lallé, situé dans le secteur sahélien strict, et le site de Kegil dans le secteur sahélo-soudanien ont les densités les plus élevées de Z. mauritiana avec respectivement 66,4 \pm 52,5 et 77,5 $\pm 61,4 \mathrm{arbres} / \mathrm{ha}$. L'analyse de la distribution des tiges par classe de diamètre montre que pour les deux espèces, les individus jeunes sont bien représentés. Cela suggère une bonne régénération de ces espèces. L'analyse de la densité et nature de régénération montre que les deux espèces se régénèrent essentiellement par drageonnage. En réponse au ramassage des fruits pour diverses utilisations, les espèces $B$. aegyptiaca et $Z$. mauritiana ont développé des stratégies alternatives de propagation en zone sahélienne. Cette aptitude à la propagation végétative permet aux deux espèces de s'adapter en zones arides caractérisées par la sécheresse et les hautes températures. 


\begin{abstract}
Wood species play an important role in the life of local communities. Among the species commonly used in Niger, are Balanites aegyptiaca and Ziziphus mauritiana. This species are exploited as food sources and as various medicinal products, with a consequent reduction in their populations. The present study aims to analyze the stand structure and the regeneration modes of the two species in two contrasting agro-ecological sectors of the Maradi region in south central Niger. A total of 60 surveys were delineated in natural formations dominated by these species and in agrosilvopastoral system in the Sahelian and Sahelo-Sudanian zones. On each tree, the diameter at $1.30 \mathrm{~m}$ from the ground, the total height and two perpendicular tree crown diameters were measured. The inventory of natural regeneration was carried out in 5 plots delineated in each plot. The mode of reproduction of the regeneration (suckers, marcots, natural seedlings) was recorded as well as the total height and the number of stems of each juvenile were. The density of $B$. aegyptiaca does not vary significantly between sites and between sectors. The Birni Lallé site, located in the strict Sahelian zone, and the Kegil site in the Sahelo-Sudanese sector stand out in terms of $Z$. mauritiana density with respectively $66.4 \pm 52.5$ and $77.5 \pm 61.4$ trees. $/ \mathrm{Ha}$. The analysis of the diameter class structure shows that for both species, young individuals are well represented. This suggests strong regeneration among these species. The analysis of the density and nature of regeneration shows that the two species regenerate mainly by suckering. In response to the collection of fruits for various uses by local populations, B. aegyptiaca and Z. mauritiana have developed alternative propagation strategies in the Sahelian zone. This ability to propagate vegetatively allows these two species to adapt to arid areas characterized by drought and high temperatures.
\end{abstract}

KEY WORDS: DISTRIBUTION OF WOODY SPECIES, B. AEGYPTIACA, Z. MAURITIANA, MARADI, NIGER, SAHELO-SUDANESE

\title{
Introduction
}

En zone sahélienne, les populations humaines ont depuis longtemps comme une de leurs principales activités la récolte de produits végétaux et la chasse pour satisfaire leurs besoins primaires. Plusieurs types de produits sont quotidiennement recherchés par les agriculteurs. Il s'agit entre autres de noix comestibles, champignons, fruits, herbes, épices, gommes, plantes aromatiques, gibier, bois, fourrage et produits végétaux ou animaux aux usages médicinaux, cosmétiques, alimentaires et/ou culturels (Sinsin et al., 2004). L'exploitation plus raisonnée et durable de ces produits pourrait contribuer non seulement à la préservation de leur diversité biologique, mais également à l'amélioration des conditions de vie de nombreuses communautés locales (FAO, 2009). Aujourd'hui, I,2 milliard de personnes, essentiellement rurales et pauvres des pays en développement, tirent en grande partie leurs ressources et revenus de plantes et animaux issus de végétations naturelles (FAO, 2010, 2012).

Ces dernières années, au Niger, l'exploitation des fruits sauvages prend de plus en plus de l'ampleur avec comme cause la baisse de la productivité des champs due à la réduction sensible de la pluviométrie et la dégradation de plus en plus prononcée des terres agricoles (Abdou, 20I4). Parmi les espèces actuellement très exploitées figurent Balanites aegyptiaca (L.) Del. de la famille des Balanitaceae et Ziziphus mauritiana Lam. de la famille des Rhamnaceae (Arbonnier, 2004; Idrissa et al., 2018).

Les fruits et autres organes de ces deux espèces sont collectés et transformés en divers produits et vendus sur les marchés de la place. Parmi les produits issus de la transformation de ces fruits on peut citer l'huile, le savon couramment vendus sur les marchés au 
Niger. Au Niger, B. aegyptiaca est traditionnellement utilisé en médecine traditionnelle pour la gestion de l'anxiété, combattre l'épilepsie, folie, jaunisse, fièvre jaune, syphilis, infestation d'helminthes, toux, et comme laxatif fort et abortif (Ojo et al., 2006; Jamilu et al., 2008; Yadav et Panghal, 2010). Plusieurs études conduites sur l'espèce ont montré que divers extraits issus de ses organes ont des effets anticonvulsivant, analgésiques et fascioliques et une activité antimicrobienne (Koko et al., 2000; Ibrahim et al., 2007; Doughari et al., 2007). L'espèce est considérée comme tolérante à la sécheresse. Elle est aussi source de nombreux métabolites secondaires ayant un potentiel de production de biocarburants à partir de l'huile (Khamis et al., 2015). Le péricarpe des fruits de Z. mauritiana est utilisé pour la fabrication d'un biscuit traditionnel (Akouri en Haoussa) et jus très prisés par les populations en zones sahélienne et sahélo-soudanienne. Ces 2 espèces assurent un apport important en nutriments et autres composés indispensables aux populations rurales (Maruza et al., 20I7). Divers composés biochimiques sont extraits du fruit de Z. mauritiana. Parmi ces produits, on peut citer les cyclopeptides, et des triterpènes, flavones et stéroïdes. Des composés aliphatiques ont été identifiés à partir des racines, des écorces et des feuilles de Z. mauritiana. Il est important de noter que certains de ces composés présentent des activités antitumorales, anti-VIH, anti-plasmodiales et anti-mycobactériennes (Wang et al., 2013).

Les décoctions des racines de Z. mauritiana sont utilisées contre la malaria (Saotoing et al., 20II). Les feuilles, les racines et l'écorce sont utilisées pour le traitement de l'hémorroïde et le panaris. L'écorce sert au tannage des cuirs et à la coloration cannelle ou grise. Elle est aussi utilisée contre les hémorragies après accouchement. Les racines et l'écorce considérées comme toxiques et purgatives deviennent violentes à fortes doses. Les racines sont également utilisées contre la syphilis, la diarrhée, la fièvre et la lèpre (Mounkaila et al., 2017; Hassane, 2008).

L'analyse de la dynamique des peuplements constitue un préalable aux actions de gestion raisonnée et durables d'aménagement et de valorisation de ces espèces en milieu naturel. Les divers prélèvements des organes de ces espèces peuvent avoir des conséquences sur l'état des peuplements naturels de ces deux espèces (Mounkaila et al., 20I7). Le ramassage des fruits entraîne une réduction de la banque séminale édaphique et peut ainsi compromettre la régénération et la dynamique de ces espèces alors qu'elles jouent un rôle économique et écologique très important pour les populations locales. Le prélèvement des écorces et les racines engendre la mortalité et déstabilise la structure des peuplements (Louppe et al., 20I8).

L'objectif principal de la présente étude est de déterminer des indicateurs d'état et la dynamique des populations de B. aegyptiaca et Z. mauritiana pour servir de base à la définition de politiques de gestion et d'utilisation durables de leurs peuplements naturels dans les zones agro-écologiques de la région de Maradi au centre sud du Niger. Il s'agit spécifiquement d'analyser (I) la structure de leurs peuplements et (2) les modes de régénération dans les formations naturelles et les systèmes agrosylvopastoraux. 


\section{Materiel et méthodes}

Zones d'étude

L'ensemble des sites ayant fait l'objet de prospections dans le cadre du présent projet de recherche sont situés dans la région de Maradi au centre sud du Niger. La région de Maradi est caractérisée par deux secteurs agro-écologiques. La partie Nord de la région se trouve dans le secteur sahélien strict et la partie sud appartient au secteur sahélosoudanien (White, I983; Saadou, I990; Ozer et Ozer, 2005).

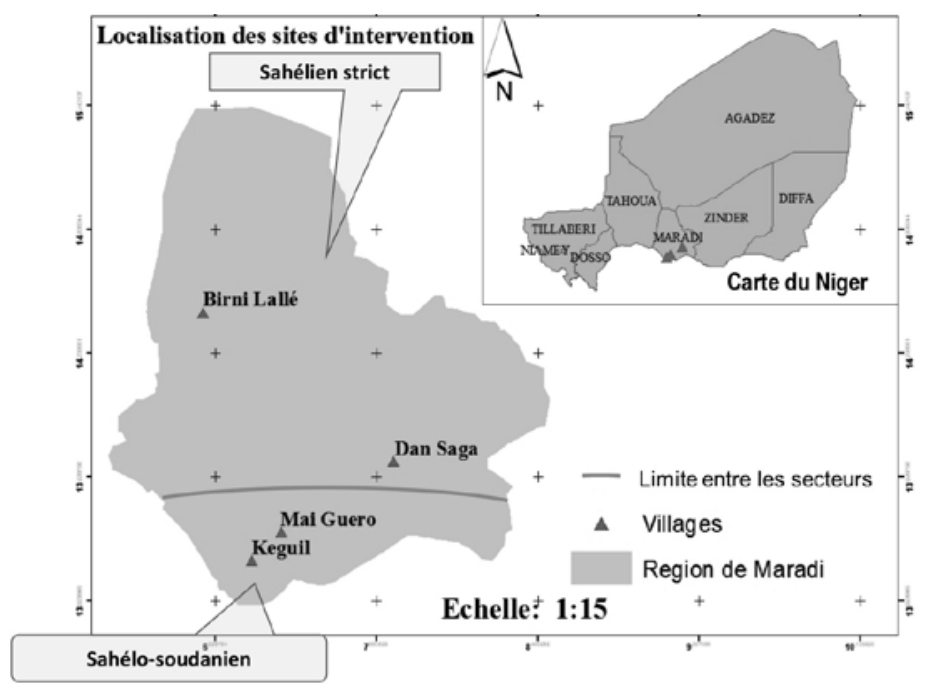

Figure 1: Localisation de la zone d'étude

Secteur sahélien strict: dans ce secteur défini comme la zone la moins arrosée (White, I983), deux sites ont fait l'objet de prospection. Les critères de choix de ces sites sont entre autres, la présence dans la zone de B. aegyptiaca et $Z$. mauritiana et aussi le fait que les femmes de ces villages ont pour activités principales, la transformation des fruits de ces espèces en huile, savon et biscuit traditionnel selon nos enquêtes préliminaires. Il s'agit du site de Dan Saga dans le département d'Aguié. Ce département se situe entre $7^{\circ} 20$ et $8^{\circ} 20$ Nord et $\mathrm{I}^{\circ} 30$ et $\mathrm{I}^{\circ} 45$ Est, et couvre une superficie de $2800 \mathrm{~km}^{2}$. Il est limité au sud par la République Fédérale du Nigeria sur une longueur de $70 \mathrm{~km}$. Le village de Dan Saga est situé à I3 km au nord de la ville d'Aguié, chef-lieu du département. Le deuxième site de ce secteur est le village de Birni Lallé dans le département de Dakoro à 90 $\mathrm{km}$ au nord de la ville de Maradi, chef-lieu de la région. Le climat de cette zone est de type sahélien caractérisé par une longue saison sèche (8 à 9 mois) d'octobre à mai suivie d'une courte saison pluvieuse ( 3 à 4 mois) de juillet à septembre (Ozer et Ozer, 2005). La pluviométrie moyenne annuelle, calculée sur les 30 dernières années, est plus élevée dans le département d'Aguié $(463,4 \pm$ I32,7 mm) que dans le département de Dakoro (36r,9 \pm Ioo $\mathrm{mm}$ ) qui se trouve à une latitude plus élevée. Dans la subdivision phytogéographique de Saadou (I990), la végétation du secteur sahélien strict est caractérisée par la présence 
de fourrés à Combretaceae sur les plateaux latéritiques, savanes sur les terrasses sableuses méridionales et steppes sur les dunes et dans les vallées sèches. La flore de la zone est dominée par des ligneux xérophytes tels que les espèces du genre Acacia, Boscia senegalensis (Pers.) Lam. Ex Poir., Balanites aegyptiaca et Ziziphus mauritiana. La strate herbacée est dominée par des thérophytes telles que Cenchrus biflorus Roxb., Schoenefeldia gracilis Kunth. et Aristida mutabilis Trin. et Rupr..

Secteur sahélo-soudanien: dans ce secteur, les sites étudiés se situent dans le département de Madarounfa (Tableau I). Il s'agit d'un premier village, Kégil, situé à $15 \mathrm{~km}$ de Madarounfa. Les prospections ont été effectuées dans les champs et dans la forêt classée de Madarounfa. Le village de Kégil fait partie des villages riverains de cette dernière forêt. Elle est classée par décret $n^{\circ} 4400$ de 1950 avec une superficie de 830 ha. Le deuxième site de ce secteur est le village de Mai Guéro, situé dans la commune de Gabi. La moyenne pluviométrique est de 552,5I mm/an (Mahamane et al., 2007). La végétation du secteur sahélo-soudanien est caractérisée par des savanes arbustive, arborées et boisées, et des fourrés à Combretaceae (Mahamane et al., 2007). Les coordonnées du centre des villages et les cumuls pluviométriques sont récapitulés dans le tableau I.

\begin{tabular}{|c|c|c|c|c|}
\hline Secteurs & Site & Latitude & Longitude & Pluviosité (mm/an) \\
\hline \multirow[t]{2}{*}{ Sahélien strict } & Birni Lallé & $\mathrm{N} 14^{\circ} 25^{\prime} 53,3^{\prime \prime}$ & E 06 $46^{\prime} 06,3^{\prime \prime}$ & $361,9 \pm 100$ \\
\hline & Dan Saga & $\mathrm{N} 13^{\circ} 42^{\prime} 12,0^{\prime \prime}$ & E $07^{\circ} 44^{\prime} 37,0^{\prime \prime}$ & $463,4 \pm 132,7$ \\
\hline \multirow[t]{2}{*}{ Sahélo-soudanien } & Kegil & $\mathrm{N} 13^{\circ} 21^{\prime} 33,8^{\prime \prime \prime}$ & E 0710'06,9" & $503,5 \pm 97,7$ \\
\hline & Mai Guéro & $\mathrm{N} 13^{\circ} 13^{\prime} 10,7^{\prime \prime}$ & E $07^{\circ} 01^{\prime} 54,0^{\prime \prime}$ & $552,51 \pm 104$ \\
\hline
\end{tabular}

Tableau 1: Caractéristiques des sites d'étude

\section{Échantillonnage}

L'échantillonnage a été effectué par le biais de transects aléatoires afin de prendre en compte la plus grande diversité du milieu (Gounot, I969). Dans les deux secteurs, seules les zones caractérisées par la présence de B. aegyptiaca et Z. mauritiana ont fait l'objet d'inventaires. Sur le site de Birni Lallé, les prospections ont été effectuées dans les champs. Dans le village de Dan Saga, les investigations ont été menées dans les champs du mil. Sur le site de Kégil, les prospections ont été effectuées dans la forêt de Madarounfa et dans les champs de mil (Pennisetum glaucum) associé au Niébé (Vigna unguculata). Sur le site de Mai Guéro, les investigations ont été effectuées dans l'aire de pâturage de Mai Guéro et dans la forêt classée de Takoudé. Sur chaque transect, des placettes ont été délimitées. Le nombre des transects par formation varie de 4 à 6 et le nombre des placettes varie de 3 à 6 par transect. Il s'agit de placettes de $1000 \mathrm{~m}^{2}$ dans les formations naturelles et $2500 \mathrm{~m}^{2}$ dans les champs conformément aux recommandations de Sun (2008). Des placeaux d'observation de la régénération de dimension $5 \mathrm{~m} \mathrm{x} 5 \mathrm{~m}\left(25 \mathrm{~m}^{2}\right)$ ont été placés dans chaque coin de la placette et un cinquième au centre (Sun, 2008). 


\section{Collecte des données}

Les paramètres dendrométriques ont fait l'objet de mesures. Dans chaque placette, tous les individus ligneux de toutes les espèces présentes de diamètre de tige supérieur ou égal à $2 \mathrm{~cm}$ ont été mesurés. Les individus jeunes de diamètre inférieur à $2 \mathrm{~cm}$ ont été considérés comme de la régénération. La hauteur totale a été mesurée à l'aide d'une perche graduée. Les diamètres des troncs à I,3 m du sol ont été mesurés (Sun, 2008) à l'aide d'un compas forestier pour les grands individus et un pied à coulisse pour les petits individus. Deux diamètres perpendiculaires du houppier ont été mesurés à l'aide d'un mètre ruban par projection verticale à la surface du sol. Au niveau des individus multicaules, un comptage systématique des rejets a été effectué. Il s'agit des jeunes tiges appelées "tiges d'avenir" issues de rejets de souche (marcottes ou drageons). Dans le cas des drageons, la distance entre le pied mère et le drageon a été mesurée afin d'estimer la distance minimale d'un drageon de son géniteur. La hauteur totale et le nombre de tiges des jeunes plantules ont été systématiquement mesurés et comptés afin de déterminer la fréquence de régénération (semis et drageons). La texture du sol appréciée par la méthode tactile (Ambouta, 1984) a été notée afin de déterminer le préférendum pédologique. Les traces d'exploitation (coupe et émondage) et l'état des individus mesurés ont été notés afin d'inventorier les indicateurs de pression.

\section{Analyse et traitement des données}

Paramètres structuraux

Les données de l'inventaire collectées sur l'ensemble des espèces et les peuplements naturels à $B$. aegyptiaca et $Z$. mauritiana ont été utilisées pour calculer les paramètres dendrométriques.

La densité est le nombre moyen d'individus sur pied estimé à l'hectare, elle se calcule par la formule :

$$
\mathrm{N}=\frac{n}{s}
$$

avec $\mathrm{n}$ : le nombre d'individus et $\mathrm{s}$ la superficie couverte en ha.

La surface terrière $\mathrm{G}$ est la somme des sections transversales de tous les arbres à $\mathrm{I}, 3 \mathrm{du}$ sol. Elle est exprimée en $\mathrm{m}^{2} /$ ha:

$$
\mathrm{G}=\frac{\grave{\mathrm{A}}}{40000 \mathrm{~s}} \sum_{\mathrm{i}=1}^{\mathrm{n}} \mathrm{d}_{\mathrm{i}}^{2}
$$

avec di : le diamètre de l'individu i en $\mathrm{cm}$ et s la superficie couverte en ha.

La contribution spécifique (Cs) est la fréquence d'une espèce (ni) sur la fréquence totale des toutes les espèces inventoriées $(\mathrm{N})$ rapportée en pourcentage.

$$
\mathrm{Cs}=\mathrm{ni}^{*} 100 / \mathrm{N}
$$


Contribution en surface terrière (GC) est la somme des surfaces terrières des individus d'une espèce (Gi) sur la somme des surfaces terrières de toutes les espèces inventoriées (Gt) rapportée en pourcentage.

$$
\mathrm{GC}=\mathrm{Gi} * 100 / \mathrm{Gt}
$$

La valeur d'importance d'une espèce (IVI) a été calculée par espèces et pour l'ensemble de formation végétale. Il permet de déterminer les espèces les plus écologiquement importantes.

$$
\text { IVI }=\text { Fr relative }(\%)+\text { Fr dominance basale }(\%)+\text { Fr dominance relative }(\%)
$$

où "Fr relative" est la fréquence relative de l'espèce, "Fr dominance basale" est la fréquence de la surface terrière de l'espèce et "Fr dominance relative" est la fréquence du recouvrement formé par l'espèce. IVI est un indice quantitatif permettant d'identifier les espèces écologiquement importantes dans une communauté végétale (Adomou et al., 2009). Il varie de o (absence de dominance) à 300 (mono-dominance).

Les paramètres dendrométriques ont été soumis aux tests afin de comparer les sites d'un même secteur et les sites de secteurs différents. Les paramètres dendrométriques des individus observés sur les différents types d'habitats et sur sols de textures différentes ont été soumis aux tests de comparaison. Les tests ont été effectués à l'aide des Logiciels R.2.I5 après vérification des conditions d'application. Il s'agit du test de Shapiro-Wilk pour la vérification de la normalité et le test d'égalité des variances pour la vérification de l'homogénéité des variances. Les données qui remplissent les conditions ont été soumises à un test paramétrique (ANOVA) et celles qui ne remplissent pas les conditions d'application des tests paramétriques ont été soumises à un test non paramétrique (Kruskall Wallis).

\section{Structure démographique}

Le logiciel Minitab r 6 a été utilisé pour estimer les paramètres de la distribution théorique de Weibull à partir des données de la distribution des classes de diamètres observées. Pour s'assurer d'un bon ajustement entre la structure observée et la distribution théorique de Weibull, une analyse log-linéaire a été effectuée à l'aide de Logiciel R.2.I5.

La distribution de Weibull à 3 paramètres (de position $a$, d'échelle ou taille $b$ et de forme c) se caractérise par une grande souplesse d'emploi et une grande variabilité de forme. Sa fonction de densité de probabilité, $f(x)$ se présente sous la forme ci-dessous (Rondeux, I999).

$$
\boldsymbol{f}(\boldsymbol{x})=\frac{c}{b}\left(\frac{x-a}{b}\right)^{c-1} \exp \left[-\left(\frac{x-a}{b}\right)^{c}\right]
$$

Où $x$ est le diamètre, la circonférence ou la hauteur des arbres et $\mathrm{f}(\mathrm{x})$ sa valeur de densité de probabilité.

Le paramètre " $a$ " correspond à la valeur seuil, c'est-à-dire à la plus petite valeur de diamètre (respectivement de hauteur) retenue pour la constitution des histogrammes. 
Le paramètre " $b$ " est lié à la valeur centrale de la distribution des classes de diamètre et de hauteur. Enfin, le paramètre "c" est lié à la structure observée et, selon sa valeur, conduit la distribution de Weibull à prendre plusieurs formes. Une analyse log-linéaire a été appliquée à l'aide de Logiciel R.2.I5 pour tester l'ajustement entre la distribution théorique de Weibull et la distribution des classes de diamètre observées.

\section{Résultats}

\section{Caractéristiques floristiques et valeurs d'importances}

L'inventaire effectué a permis de recenser 28 espèces ligneuses réparties en 12 familles et 22 genres dont le plus représenté est Acacia avec 5 espèces. La famille la plus représentée dans les deux secteurs est celle des Fabaceae avec 4 espèces à Birni Lallé, 7 espèces à Dan Saga et à Kegil et enfin 5 espèces à Mai Guéro. Les familles des Balanitaceae et Rhamnaceae sont présentes dans tous les secteurs avec un représentant en l'occurrence B. aegyptiaca pour la famille des Balanitaceae et deux espèces Z. mauritiana et Z. spina-christi, pour la famille des Rhamnanceae. L'analyse de l'indice des valeurs d'importance montre que les espèces les plus représentées dans l'ensemble des relevés effectués aussi bien dans le secteur sahélien strict qu'en secteur sahélo-soudanien sont $\mathrm{B}$. aegytiaca avec un indice de 88 pour Birni Lallé, I90,3 pour Dan Saga, I07,8 pour Kegil et 73,9 à Mai Guéro, suivie de Z. mauritiana avec I70,5 pour Birni Lallé, 6, I pour Dan Saga, 68,6 pour Kegil et 73,9 pour 300 à Mai Guéro (Tableau 2). 


\begin{tabular}{|c|c|c|c|c|c|}
\hline \multirow[t]{2}{*}{ Familles } & \multirow[t]{2}{*}{ Espèces } & \multicolumn{2}{|l|}{ Sahel Strict } & \multicolumn{2}{|c|}{ Sahélo- soudanien } \\
\hline & & Birni Lallé & Dan Saga & Kegil & Mai Guéro \\
\hline \multirow[t]{2}{*}{ Anacardiaceae } & Lannea microcarpa & 0,0 & 7,9 & 0,0 & 0,0 \\
\hline & Sclerocarya birrea & 0,0 & 4,0 & 0,6 & 0,0 \\
\hline Annonaceae & Annona senegalensis & 0,0 & 9,1 & 3,7 & 1,4 \\
\hline Balanitaceae & Balanites aegyptiaca & 88,0 & 190,3 & 107,8 & 73,9 \\
\hline Malvaceae & Adansonia digitata & 0,0 & 0,6 & 0,0 & 6,3 \\
\hline Burseraceae & Commiphora africana & 0,0 & 2,0 & 0,0 & 0,0 \\
\hline \multirow[t]{2}{*}{ Capparaceae } & Maerua angolensis & 0,0 & 0,4 & 0,0 & 0,0 \\
\hline & Maerua crassifolia & 0,0 & 3,0 & 4,2 & 0,0 \\
\hline \multirow[t]{3}{*}{ Combretaceae } & Anogeisus leiocarpus & 0,0 & 0,0 & 4,7 & 0,0 \\
\hline & Combretum glutinosum & 0,0 & 11,2 & 0,6 & 0,0 \\
\hline & Combretum micranthum & 0,0 & 0,0 & 3,5 & 0,8 \\
\hline Ebenaceae & Diospyros mespilifornis & 0,0 & 0,0 & 3,3 & 9,3 \\
\hline \multirow[t]{11}{*}{ Fabaceae } & Acacia ataxacantha & 0,0 & 0,0 & 0,6 & 0,0 \\
\hline & Acacia nilotica & 25,1 & 5,8 & 34,0 & 57,5 \\
\hline & Acacia raddiana & 12,7 & 0,0 & 0,0 & 0,0 \\
\hline & Acacia senegal & 0,0 & 0,5 & 0,0 & 18,2 \\
\hline & Acacia sieberiana & 0,0 & 0,0 & 0,5 & 0,0 \\
\hline & Bauhinia rufescens & 0,6 & 1,6 & 12,8 & 2,9 \\
\hline & Cassia singueana & 0,0 & 0,5 & 0,0 & 0,0 \\
\hline & Faidherbia albida & 3,3 & 26,9 & 4,7 & 37,7 \\
\hline & Piliostigma reticulatum & 0,0 & 19,9 & 45,9 & 44,5 \\
\hline & Prosopis africana & 0,0 & 4,0 & 0,0 & 0,0 \\
\hline & Prosopis juliflora & 0,0 & 0,0 & 4,6 & 0,0 \\
\hline Meliaceae & Azadirachta indica & 0,0 & 6,3 & 0,0 & 2,8 \\
\hline Moraceae & Ficus platyphylla & 0,0 & 0,0 & 0,0 & 0,7 \\
\hline Rhamnaceae & Ziziphus mauritiana & 170,5 & 6,1 & 68,6 & 41,2 \\
\hline Rhamnaceae & Ziziphus spina-christi & 0,0 & 0,0 & 0,0 & 1,9 \\
\hline Rubiaceae & Mitragyna inermis & 0,0 & 0,0 & 0,0 & 0,8 \\
\hline \multicolumn{2}{|l|}{ Total } & 300 & 300 & 300 & 300 \\
\hline
\end{tabular}

Tableau 2: Indices de valeurs d'importance

Caractéristiques structurales globales

Les sites du secteur sahélo-soudanien se distinguent significativement des sites du secteur sahélien strict par des densités d'adultes plus élevées. La densité est de 333,I士Ior,7 pieds/ ha à Kegil et I94,6士IIo,4 pieds/ha à Mai Guéro. Par contre, la différence des densités n'est pas significative entre Birni Lallé et Dan Saga qui sont tous les deux du secteur sahélien 
strict. Le diamètre moyen le plus élevé est observé au niveau du site de Birni Lallé avec

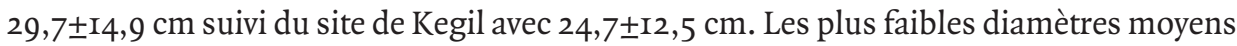
et hauteurs totales moyennes sont observés au niveau du site de Dan Saga dans le secteur sahélien strict (Tableau 3). La densité des juvéniles est plus élevée au niveau du site de Mai Guéro situé en zone sahélo-soudanienne suivi du site de Birni Lallé dans le secteur sahélien. La plus faible densité est observée au niveau du site de Dan Saga situé dans la même zone. La densité élevée observée au niveau du site de Kegil se traduit par une surface terrière élevée. La hauteur totale moyenne la plus élevée est observée au niveau du site de Mai Guéro.

\begin{tabular}{|l|l|l|l|l|l|}
\hline \multirow{2}{*}{$\begin{array}{l}\text { Paramètres } \\
\text { structuraux }\end{array}$} & \multicolumn{2}{|l|}{ Sahel Strict } & \multicolumn{2}{l|}{ Sahélo-soudanien } & P-value \\
\cline { 2 - 6 } & Birni Lallé & Dan Saga & kegil & Mai Guéro & \\
\hline $\begin{array}{l}\text { Densité adulte } \\
(\mathrm{N}, \text { pieds/ha) }\end{array}$ & $150(57,9)^{\mathrm{a}}$ & $153(62,4)^{\mathrm{a}}$ & $333,1(101,7)^{\mathrm{b}}$ & $194,6(110,4)^{\mathrm{a}}$ & $<0,001^{*}$ \\
\hline $\begin{array}{l}\text { Diamètre moyen } \\
(\mathrm{D}, \mathrm{cm})\end{array}$ & $29,73(14,9)^{\mathrm{a}}$ & $18,4(14,7)^{\mathrm{b}}$ & $24,7(12,5)^{\mathrm{c}}$ & $23,16(14,8)^{\mathrm{c}}$ & $<0,001^{*}$ \\
\hline $\begin{array}{l}\text { Surface terrière } \\
\left(\mathrm{G}, \mathrm{m}^{2} / \text { ha) }\right.\end{array}$ & $14,3(8,4)^{\mathrm{a}}$ & $6,9(2,5)^{\mathrm{b}}$ & $21,05(8,74)^{\mathrm{c}}$ & $13,5(3,95)^{\mathrm{a}}$ & $<0,001^{*}$ \\
\hline $\begin{array}{l}\text { Hauteur moyenne } \\
(\mathrm{H}, \mathrm{m})\end{array}$ & $7,8(6,8)^{\mathrm{a}}$ & $4,5(2,9)^{\mathrm{b}}$ & $7,5(3,03)^{\mathrm{a}}$ & $8,9(3,4)^{\mathrm{c}}$ & $<0,001^{*}$ \\
\hline $\begin{array}{l}\text { Densité juvéniles } \\
(\mathrm{Nr}, \text { plants/ha) }\end{array}$ & $342,8(338,5)^{\mathrm{a}}$ & $181,3(129,9)^{\mathrm{a}}$ & $285(323,9)^{\mathrm{a}}$ & $378,6(342,9)^{\mathrm{a}}$ & 0,302 \\
\hline $\begin{array}{l}\text { Densité totale } \\
(\mathrm{N}, \text { pieds/ha) }\end{array}$ & $492,8(395,9)^{\mathrm{a}}$ & $334,3(192,3)^{\mathrm{c}}$ & $618,1(425,6)^{\mathrm{b}}$ & $573,2(453,3)^{\mathrm{a}}$ & $<0,001^{*}$ \\
\hline $\begin{array}{l}\text { Taux de } \\
\text { régénération (\%) }\end{array}$ & 69,6 & 54,2 & 46,1 & 65,9 & - \\
\hline
\end{tabular}

Tableau 3: Paramètres structuraux globaux des peuplements des sites et secteurs d'étude

Sur une même ligne du tableau les cases ayant les mêmes lettres ne sont pas significativement différentes. Les valeurs moyennes sont accompagnées de l'écart type.

\section{Caractéristiques structurales de $B$. aegyptica}

La densité de B. aegyptiaca la plus élevée est observée au niveau du site de Kegil, situé dans le secteur sahélo-soudanien avec une densité de IIO \pm I2I,4 pieds/ha suivi du site de Dan Saga avec une densité de $84 \pm 43,8$ pieds/ha. La plus faible densité des adultes est observée au niveau du site de Mai Guéro dans le secteur sahélo-soudanien. Les individus de B. aegyptiaca les plus vigoureux sont observés au niveau de Kegil avec un diamètre moyen de $28,9 \pm \mathrm{II}, 2 \mathrm{~cm}$ et une hauteur totale moyenne de $7,4 \pm 2, \mathrm{I}$. Les plus faibles valeurs sont observées au niveau des sites de Dan Saga et de Birni Lallé. La densité des plantules la plus

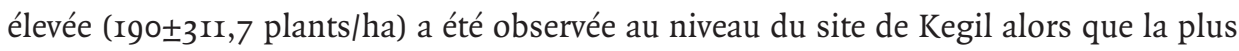
faible a été observée au niveau du site de Mai Guéro (90,6士I8I, I plants/ha) (Tableau 4). 


\begin{tabular}{|c|c|c|c|c|c|}
\hline \multirow[t]{2}{*}{ Paramètres structuraux } & \multicolumn{2}{|l|}{ Sahel Strict } & \multicolumn{2}{|c|}{ Sahélo-soudanien } & \multirow[t]{2}{*}{ P-value } \\
\hline & Birni Lallé & Dan Saga & kegil & Mai Guéro & \\
\hline $\begin{array}{l}\text { Densité adulte } \\
\text { (NBal, pieds/ha) }\end{array}$ & $65,7(56,9)^{a}$ & $84(43,8)^{a}$ & $110(121,4)^{\mathrm{a}}$ & $51,3(89,9)^{a}$ & 0,177 \\
\hline $\begin{array}{l}\text { Diamètre moyen } \\
\text { (DBal, cm) }\end{array}$ & $22,1(12,8)^{\mathrm{a}}$ & $21,6(14,2)^{a}$ & $28,9(11,2)^{b}$ & $22,6(12,7)^{\mathrm{a}}$ & $<0,001^{*}$ \\
\hline $\begin{array}{l}\text { Surface terrière } \\
\text { (GBal, m²/ha) }\end{array}$ & $3,4(3,4)^{a}$ & $4,5(2,6)^{a}$ & $8,8(9,7)^{b}$ & $3,1(5,4)^{a}$ & $0,035^{*}$ \\
\hline $\begin{array}{l}\text { Hauteur moyenne } \\
(\mathrm{HBal}, \mathrm{m})\end{array}$ & $6,91(9,7)^{\mathrm{a}}$ & $5,01(2,6)^{b}$ & $7,4(2,1)^{a}$ & $8,4(3,5)^{c}$ & $<0,001^{*}$ \\
\hline $\begin{array}{l}\text { Densité juvénile } \\
\text { (NrBal plants/ha) }\end{array}$ & $182,8(270,7)^{\mathrm{a}}$ & $101,3(122,6)^{\mathrm{a}}$ & $190(311,7)^{\mathrm{a}}$ & $90,6(181,1)^{a}$ & 0,524 \\
\hline $\begin{array}{l}\text { Contribution densité adulte } \\
\text { (Nc\%) }\end{array}$ & 13,3 & 25,1 & 17,8 & 8,9 & - \\
\hline $\begin{array}{l}\text { Contribution densité juvénile } \\
\text { (NrBal \%) }\end{array}$ & 37,2 & 30,3 & 30,7 & 15,8 & - \\
\hline $\begin{array}{l}\text { Contribution en surface térrière } \\
(\mathrm{GC}, \%)\end{array}$ & 23,8 & 65,2 & 41,8 & 22,9 & - \\
\hline
\end{tabular}

Tableau 4: Paramètres structuraux des populations de B. aegyptiaca

Sur une même ligne du tableau les cases ayant les mêmes lettres ne sont pas significativement différentes. Les valeurs moyennes sont accompagnées de l'écart type.

\section{Caractéristiques structurales de Z. mauritiana}

Le site de Dan Saga se singularise par sa faible densité des adultes de Z. mauritiana avec seulement 5,3 \pm Io, 6 pieds/ha alors que le site de Mai Guéro présente une densité de 26 $\pm 43,5$ pieds/ha. La densité la plus significativement élevée est observée au niveau du site de Kegil avec 77,5 \pm 6 I, 4 pieds/ha suivi du site de Birni Lallé $(66,4 \pm 52,5$ pieds/ha). Par contre, les sites du secteur sahélo-soudanien se distinguent par des densités de plantules

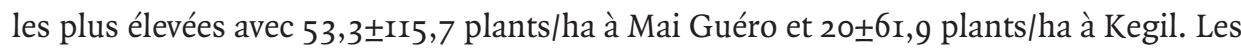
individus de $\mathrm{Z}$. mauritiana les plus vigoureux sont observés au niveau du site de Birni Lallé avec un diamètre moyen de 35, I $\pm 13,6 \mathrm{~cm}$ et une hauteur totale de $8,5 \pm 2,8 \mathrm{~m}$. Ce dernier site est suivi de Kegil dans le secteur sahélo-soudanien qui se caractérise par des individus avec un diamètre moyen de $24,2 \pm 9, \mathrm{I} \mathrm{cm}$ et $8,06 \pm 3$,oI $\mathrm{m}$ de hauteur totale. Le peuplement de Z. mauritiana du site de Dan Saga est dominé par de petits individus avec des hauteurs totales et des diamètres faibles. Les densités de plantules de Z. mauritiana les plus élevées ont été observées au niveau des sites du secteur sahélo-soudanien (Tableau 5). 


\begin{tabular}{l|l|l|l|l|l|}
\hline \multirow{2}{*}{ Paramètres structuraux } & \multicolumn{2}{l|}{ Sahel Strict } & \multicolumn{2}{l|}{ Sahélo-soudanien } & \multirow{2}{*}{ P-value } \\
\cline { 2 - 5 } & Birni Lallé & Dan Saga & kegil & Mai Guéro & \\
\hline $\begin{array}{l}\text { Densité adulte } \\
\text { (NZiz, pieds/ha) }\end{array}$ & $66,4(52,5)^{\mathrm{a}}$ & $5,3(10,6)^{\mathrm{c}}$ & $\begin{array}{l}77,5 \\
(61,4)^{\mathrm{a}}\end{array}$ & $26(43,5)^{\mathrm{a}}$ & $<0,001^{*}$ \\
\hline $\begin{array}{l}\text { Diamètre moyen } \\
\text { (DZiz, cm) }\end{array}$ & $35,1(13,6)^{\mathrm{a}}$ & $9,9(6,1)^{\mathrm{b}}$ & $24,2(9,1)^{\mathrm{c}}$ & $22,3(10,7)^{\mathrm{d}}$ & $<0,001^{*}$ \\
\hline $\begin{array}{l}\text { Surface terrière } \\
\text { (GZiz, m²/ha) }\end{array}$ & $8,6(7,2)^{\mathrm{a}}$ & $0,06(0,1)^{\mathrm{b}}$ & $4,6(4,3)^{\mathrm{c}}$ & $1,3(1,7)^{\mathrm{d}}$ & $<0,001^{*}$ \\
\hline $\begin{array}{l}\text { Hauteur moyenne } \\
\text { (HZiz, m) }\end{array}$ & $8,5(2,8)^{\mathrm{a}}$ & $3,06(0,9)^{\mathrm{b}}$ & $\begin{array}{l}8,06 \\
(3,01)^{\mathrm{a}}\end{array}$ & $8,5(2,8)^{\mathrm{a}}$ & $<0,001^{*}$ \\
\hline $\begin{array}{l}\text { Densité juvénile } \\
\text { (NrZiz plants/ha) }\end{array}$ & $1,2(1,3)^{\mathrm{b}}$ & $2,1(3,2)^{\mathrm{b}}$ & $20(61,9)^{\mathrm{a}}$ & $53,3(115,7)^{\mathrm{c}}$ & $<0,001^{*}$ \\
\hline $\begin{array}{l}\text { Contribution densité adulte } \\
\text { (Nc\%) }\end{array}$ & 13,5 & 1,6 & 12,5 & 4,5 & - \\
\hline $\begin{array}{l}\text { Contribution densitéjuvénile } \\
\text { (NrZiz \%) }\end{array}$ & 0,2 & 0,6 & 3,2 & 9,3 & - \\
\hline $\begin{array}{l}\text { Contribution en surface térrière } \\
\text { (GC, \%) }\end{array}$ & 60,1 & 0,9 & 21,9 & 9,6 & - \\
\hline
\end{tabular}

Tableau 5: Paramètres structuraux des populations de Z. mauritiana

Sur une même ligne du tableau les cases ayant les mêmes lettres ne sont pas significativement différentes. Les valeurs moyennes sont accompagnées de l'écart type

Impact de la texture du sol et du type d'habitat sur les caractéristiques structurales de B. aegyptiaca

Les sols argileux se distinguent significativement du sol sableux par des individus de diamètre moyen du tronc élevé. Une différence hautement significative a été observée entre les hauteurs moyennes des peuplements sur sols de différentes textures $(p<0,001)$. Des différences significatives ont été également observées au niveau des moyennes des densités, de diamètres et des hauteurs totales. Les individus les plus vigoureux ont été observés au niveau des aires de pâturage et de la forêt classée du secteur sahélo-soudanien (Tableau 6).

\begin{tabular}{l|l|l|l|l|l|l|l}
\hline \multirow{2}{*}{$\begin{array}{l}\text { Paramètres } \\
\text { structuraux }\end{array}$} & \multicolumn{4}{l}{ Texture du sol } & \multicolumn{2}{l}{ Type de formation } \\
\cline { 2 - 8 } & Sableux & Argileux & P-value & Champs & $\begin{array}{l}\text { Aire de } \\
\text { pâturage }\end{array}$ & $\begin{array}{l}\text { Forêt } \\
\text { classée }\end{array}$ & P-Value \\
\hline Densité $($ Pieds/ha) & $67,3(32,3)^{\mathrm{a}}$ & $80,6(65,2)^{\mathrm{a}}$ & 0,483 & $99,3(66)^{\mathrm{a}}$ & $58,5(51,6)^{\mathrm{c}}$ & $23(10,05)^{\mathrm{b}}$ & $0,004^{*}$ \\
\hline Diamètre moyen $(\mathrm{cm})$ & $22,2(13,7)^{\mathrm{a}}$ & $26,9(11,9)^{\mathrm{b}}$ & $<0,001^{*}$ & $24,6(12,8)^{\mathrm{a}}$ & $21,9(12,2)^{\mathrm{a}}$ & $28,4(15,9)^{\mathrm{b}}$ & $0,026^{*}$ \\
\hline Hauteur moyenne $(\mathrm{m})$ & $5,3(2,7)^{\mathrm{a}}$ & $7,7(2,7)^{\mathrm{a}}$ & $<0,001^{*}$ & $5,9(2,8)^{\mathrm{a}}$ & $8,1(3,8)^{\mathrm{b}}$ & $8,2(3,2)^{\mathrm{b}}$ & $<0,001^{*}$ \\
\hline
\end{tabular}

Tableau 6: Impact de la texture du sol et du type d'habitat sur les caractéristiques structurales de $B$. aegyptiaca

Sur une même ligne du tableau les cases ayant les mêmes lettres ne sont pas significativement différentes. Les valeurs moyennes sont accompagnées de l'écart type 
Impact de la texture du sol et du type d'habitat sur les caractéristiques structurales de Z. mauritiana

Les peuplements de Z. mauritiana sont plus denses sur sol argileux avec $67,6 \pm 47,2$ pieds/ ha contre $28,7 \pm 22,6$ pieds/ha sur sol sableux. La hauteur moyenne est significativement plus élevée au niveau des peuplements sur sols argileux avec $8,3 \pm 2,8 \mathrm{~m}$ alors qu'elle est de $7,3 \pm 3,8 \mathrm{~m}$ pour les individus sur sols sableux. Il n'existe pas de différences significatives entre les densités des peuplements de Z. mauritiana observées dans les différents types de formation. Par contre, des différences significatives ont été observées au niveau des diamètres du tronc et hauteurs totales. Le diamètre moyen le plus élevé est observé au niveau des individus des peuplements des champs avec 29,3 $\pm 13,9 \mathrm{~cm}$ suivis des individus des aires de pâturage avec 28,8 \pm I5, I cm. Par contre, la hauteur moyenne des individus la plus élevée avec 9,2 \pm I,6 $\mathrm{m}$ a été observée dans les aires de pâturage alors que la plus faible est observée au niveau des champs avec 7,I \pm 2,9 $\mathrm{m}$ (Tableau 7).

\begin{tabular}{l|l|l|l|l|l|l|l}
\hline \multirow{2}{*}{$\begin{array}{l}\text { Paramètres } \\
\text { structuraux }\end{array}$} & \multicolumn{4}{l}{ Texture du sol } & \multicolumn{2}{l}{ Type de formation } \\
\cline { 2 - 8 } & Sableux & Argileux & P-value & Champs & $\begin{array}{l}\text { Aire de } \\
\text { pâturage }\end{array}$ & $\begin{array}{l}\text { Forêt } \\
\text { classée }\end{array}$ & P-Value \\
\hline Densité (pieds/ha) & $28,7(22,6)^{\mathrm{a}}$ & $67,6(47,2)^{\mathrm{b}}$ & $0,041^{*}$ & $44,5(26,2)^{\mathrm{a}}$ & $45(27,8)^{\mathrm{a}}$ & $49,9(24,9)^{\mathrm{a}}$ & 0,949 \\
\hline Diamètre moyen $(\mathrm{cm})$ & $28,5(16,2)^{\mathrm{a}}$ & $26,7(11,8)^{\mathrm{a}}$ & 0,398 & $29,3(13,9)^{\mathrm{a}}$ & $28,8(15,1)^{\mathrm{a}}$ & $24,3(9,8)^{\mathrm{b}}$ & $0,01^{*}$ \\
\hline Hauteur moyenne $(\mathrm{m})$ & $7,3(3,8)^{\mathrm{a}}$ & $8,3(2,8)^{\mathrm{b}}$ & $0,03^{*}$ & $7,1(2,9)^{\mathrm{a}}$ & $9,2(1,6)^{\mathrm{b}}$ & $8,3(3,3)^{\mathrm{b}}$ & $0,001^{*}$ \\
\hline
\end{tabular}

Tableau 7: Impact de la texture du sol et du type d'habitat sur les caractéristiques structurales de $Z$. mauritiana

Sur une même ligne du tableau les cases ayant les mêmes lettres ne sont pas significativement différentes. Les valeurs moyennes sont accompagnées de l'écart type

\section{Caractéristiques de la régénération}

L'analyse des nombres de tiges par touffe de régénération ne révèle pas de différence significative entre les sites. Les hauteurs des plantules de B. aegytiaca sont plus élevées dans le secteur sahélien strict $(\mathrm{p}<0,00 \mathrm{I})$. Cette tendance est inversée au niveau des $\mathrm{Z}$. mauritiana où la hauteur des plantules est plus élevée dans le secteur sahélo-soudanien ( $\mathrm{p}=$ o,oor) (Tableau 8). 


\begin{tabular}{|c|c|c|c|c|c|c|c|}
\hline \multirow[t]{2}{*}{ Espèces } & \multirow{2}{*}{\multicolumn{2}{|c|}{ Paramètres }} & \multicolumn{2}{|c|}{ Sahel Strict } & \multicolumn{2}{|c|}{ Sahélo-soudanien } & \multirow[t]{2}{*}{ P-value } \\
\hline & & & BirniLallé & Dan saga & Kegil & Mai Guéro & \\
\hline \multirow{6}{*}{ 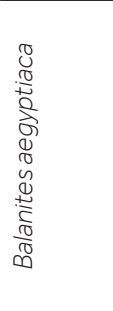 } & \multicolumn{2}{|c|}{ Nombre de tige par plantule } & $5,1(4,3)^{a}$ & $6,3(8,4)^{\mathrm{a}}$ & $5,7(6,6)^{a}$ & $3,2(1,3)^{\mathrm{a}}$ & 0,401 \\
\hline & \multicolumn{2}{|c|}{ Hauteur dominante (m) } & $2,7(1,6)^{a}$ & $3,2(2)^{c}$ & $1,2(0,6)^{b}$ & $1,9(1,2)^{b}$ & $<0,001^{*}$ \\
\hline & \multirow[t]{2}{*}{ Nature } & Semis (\%) & 25,0 & 36,8 & 31,6 & 23,5 & - \\
\hline & & Drageon (\%) & 75,0 & 63,2 & 68,4 & 76,5 & \\
\hline & \multicolumn{2}{|c|}{$\begin{array}{l}\text { Distance moyenne de } \\
\text { drageon }(\mathrm{m})\end{array}$} & $3,4(1,2)^{a}$ & $6,3(4,7)^{\mathrm{a}}$ & $4,3(3,9)^{a}$ & $5,1(1,2)^{\mathrm{a}}$ & 0,388 \\
\hline & \multicolumn{2}{|c|}{ Nombre de rejets } & $17(17,9)^{\mathrm{a}}$ & $19,3,8(13,4)^{a}$ & $10,8(13,3)^{\mathrm{a}}$ & $8,8(5,5)^{a}$ & 0,375 \\
\hline \multirow{6}{*}{ 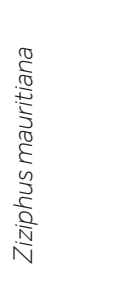 } & \multicolumn{2}{|c|}{ Nombre de tige par plantule } & $1,2(1,39)^{a}$ & $1,2(1,7)^{\mathrm{a}}$ & $6(2,9)^{b}$ & $4,5(2,8)^{b}$ & $0,003^{*}$ \\
\hline & \multicolumn{2}{|c|}{ Hauteur dominante } & $1,9(1,6)^{\mathrm{a}}$ & $1,1(1,7)^{\mathrm{a}}$ & $2,5(0,4)^{b}$ & $6,6(4,3)^{b}$ & $0,001^{*}$ \\
\hline & \multirow[t]{2}{*}{ Nature } & Semis (\%) & 65 & 46 & 25 & 40 & \\
\hline & & Drageon (\%) & 35 & 54 & 75 & 60 & \\
\hline & \multicolumn{2}{|c|}{$\begin{array}{l}\text { Distance moyenne de } \\
\text { drageon }(\mathrm{m})\end{array}$} & $1,3(1,3)^{\mathrm{a}}$ & $1,9(1,6)^{\mathrm{a}}$ & $1,9(1,3)^{\mathrm{a}}$ & $0,8(0,4)^{\mathrm{a}}$ & 0,125 \\
\hline & \multicolumn{2}{|c|}{ Nombre de rejets } & $44,8(52,5)^{a}$ & $7,3(8,3)^{b}$ & $14,5(16,06)^{c}$ & $13,3(9,3)^{c}$ & $0,002 *$ \\
\hline
\end{tabular}

Tableau 8: Structure des plantules issues de la régénération

Sur une même ligne du tableau les cases ayant les mêmes lettres ne sont pas significativement différentes. Les valeurs moyennes sont accompagnées de l'écart type

Indices des pressions anthropiques sur les individus des deux espèces

La fréquence de coupe est plus élevée à Mai Guéro alors qu'elle est plus faible à Birni Lallé dans le secteur sahélien strict aussi bien sur B. aegyptiaca que sur Z. mauritiana. D'un point de vue morphologique, une fréquence élevée des individus penchés est observée au niveau de Z. mauritiana surtout dans le secteur sahélien strict avec $42,3 \%$ et $37,5 \%$ respectivement à Birni Lallé et Dan Saga contre 22,58\% à Kegil et I8,9\% à Mai Guéro (Tableau 9).

\begin{tabular}{l|l|r|r|r|r}
\hline \multirow{2}{*}{ Espèces } & \multirow{2}{*}{ Facteurs } & \multicolumn{2}{|l|}{ Sahel Strict } & \multicolumn{2}{l}{ Sahélo-soudanien } \\
\cline { 3 - 7 } & & \multicolumn{1}{l}{ Birni lallé } & Dan Saga & kegil & \multicolumn{1}{c}{ Mai Guéro } \\
\hline \multirow{3}{*}{ Balanites aegyptiaca } & Coupé (\%) & 3,50 & 2,50 & 3,97 & 16,88 \\
\cline { 2 - 6 } & Penché (\%) & 9,78 & 3,17 & 3,98 & 6,60 \\
\cline { 2 - 6 } & Emondé (\%) & 2,30 & 6,40 & 8,50 & 8,50 \\
\hline \multirow{2}{*}{ Ziziphus mauritiana } & Coupé (\%) & 3,00 & 1,20 & 4,84 & 10,81 \\
\cline { 2 - 6 } & Penché (\%) & 42,39 & 37,50 & 22,58 & 18,92 \\
\cline { 2 - 6 } & Emondé (\%) & 2,00 & 2,50 & 4,84 & 3,10 \\
\hline
\end{tabular}

Tableau 9: Facteurs anthropiques et morphologique 


\section{Structure en classe de diamètre des peuplements de B. aegyptiaca}

L'analyse des structures en classes des diamètres des populations de B. aegyptiaca montre des tendances variables en fonction des sites et des secteurs. Sur les sites du secteur sahélien strict, les individus jeunes de classes de diamètres compris entre 2 à Io $\mathrm{cm}$ sont relativement les plus représentés soit $44,3 \%$ de l'ensemble d'individus de la population de Birni Lallé et 53,2\% à Dan Saga, les individus adultes de diamètres supérieurs à $38 \mathrm{~cm}$ sont faiblement présents. Les structures de ces populations montrent une distribution plus ou moins stable avec des paramètres de forme $c$ de la distribution théorique de Weibull proche de I, caractéristiques des peuplements mono-spécifiques avec prédominance d'individus jeunes. Dans le secteur sahélo-soudanien les populations de B. aegyptiaca sont plus perturbées. Les individus jeunes et les individus adultes sont faiblement représentés. Les individus prédominants de l'ensemble d'individus de la population dans ce secteur sont de classes de diamètres compris entre $\mathrm{I} 8$ et $26 \mathrm{~cm}$ soit $35,2 \%$ à Kegil et de 6 à $\mathrm{I} 4 \mathrm{~cm}$ soit 37,2\% à Mai Guéro. Globalement, dans le secteur sahélo-soudanien, la structure en classes de diamètre suit une distribution en cloche avec des paramètres de forme c relative à la distribution théorique de Weibull supérieur à I (Figure 2). Pour l'ensemble des structures, les distributions des classes de diamètre observées s'ajustent à la distribution théorique de Weibull avec un paramètre $(\mathrm{P}<0,05)$.
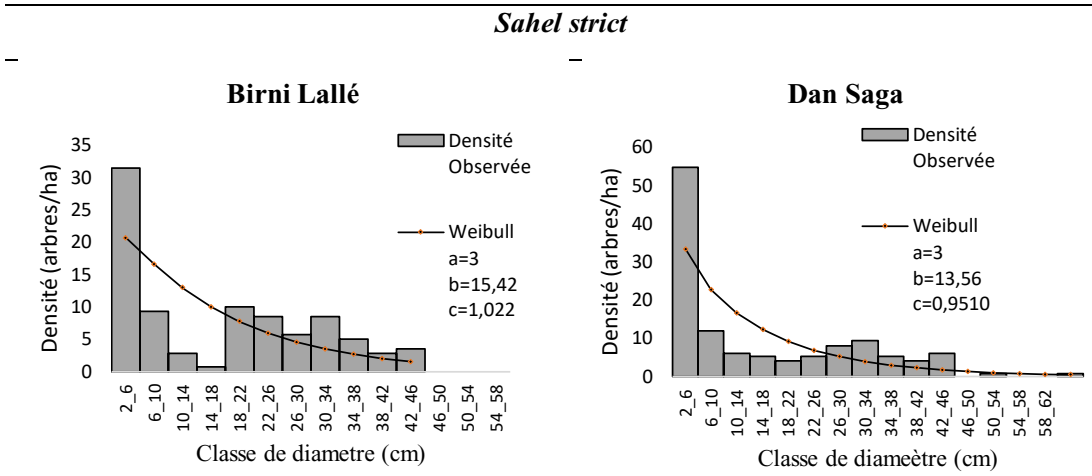

Sahélo-soudanien
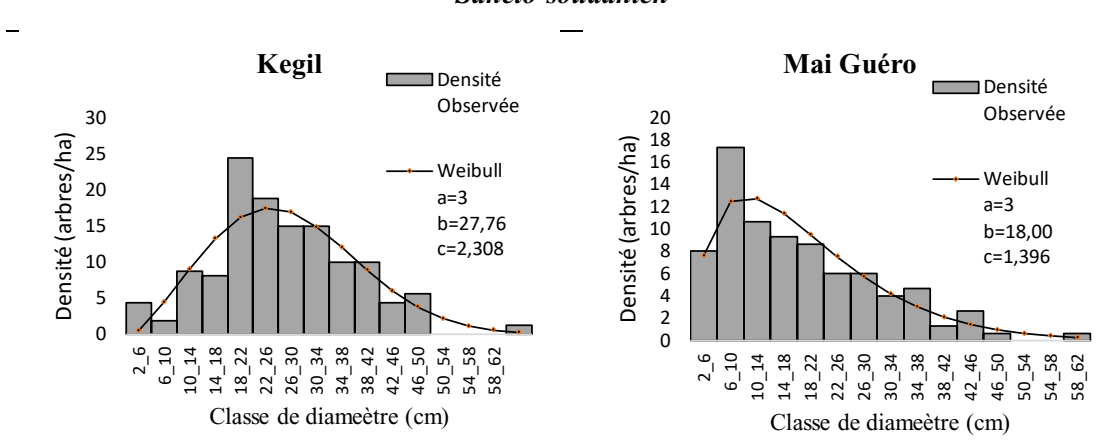

Figure 2: Structure en classe de diamètre de B. aegyptiaca 


\section{Structure des peuplements de Z. mauritiana}

L'analyse de la distribution des classes de diamètres montre que les individus des populations de Z. mauritiana présente une structure relativement perturbée presque dans tous les secteurs. En effet, dans le secteur sahélien strict les individus jeunes et adultes sont largement moins représentés. Les individus les plus représentés sont de classe de diamètre compris entre 18 et $38 \mathrm{~cm}$ soit $60,9 \%$ de l'ensemble des individus de la population de Birni Lallé et de 2 à $6 \mathrm{~cm}$ soit $69,2 \%$ des populations de Dan Saga. Dans le secteur sahélo-soudanien où la densité de Z. mauritiana est un peu plus importante, la distribution des classes de diamètre n'est pas meilleure que de celle du secteur sahélien strict. Les individus de classe de diamètres compris entre I4 et $34 \mathrm{~cm}$ dominent le site de Kegil soit 69,4\% d'individus de la population. Sur le site de Mai Guéro, il a été constaté une distribution bimodale dont le premier mode correspond aux individus jeunes de classe de diamètre compris entre 2 et $6 \mathrm{~cm}$, soit 23,4\% et le deuxième mode est dominé par des individus de classe de diamètre compris entre I4 et $26 \mathrm{~cm}$, soit $42,18 \%$ des individus de la population. La structure en classes de diamètres suit une distribution en cloche avec des paramètres de forme $\mathrm{c}$ de la distribution théorique de Weibull supérieur à I pour tous les sites (Figure 3). Pour l'ensemble des structures, les distributions des classes de diamètre observées s'ajustent à la distribution théorique de Weibull avec un paramètre $(\mathrm{P}<0,05)$.
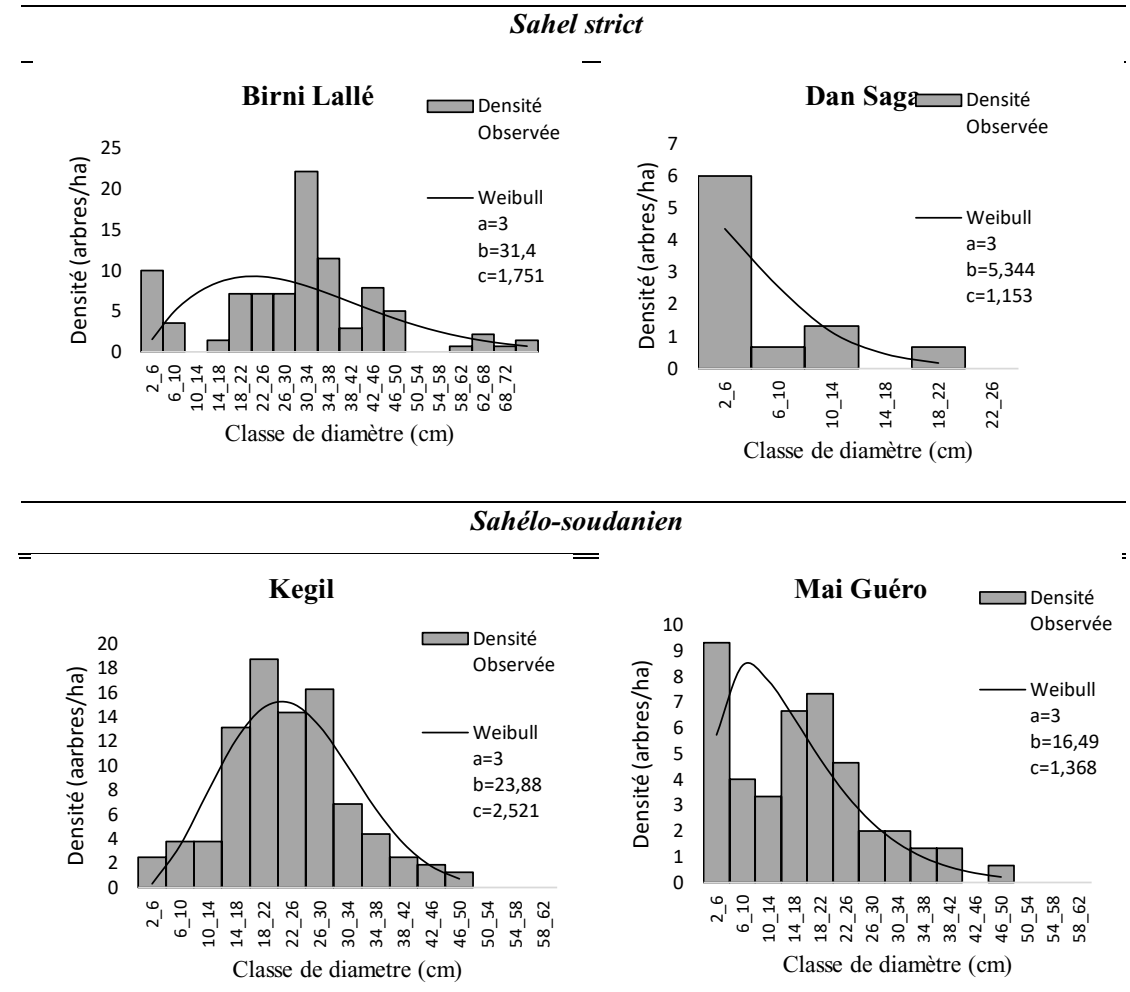

Figure 3: Structure en classe de diamètre de Ziziphus mauritiana 


\section{Discussion}

En zone aride telle que la zone sahélienne, la flore ligneuse est relativement très peu diversifiée avec comme cause les conditions très difficiles d'installation et de développement de la végétation (Deblauwe, 2010). Le cumul pluviométrique annuel très faible, la forte pression anthropique et pastorale, sont entre autres, des facteurs limitant l'épanouissement de la végétation (Ozer et Ozer, 2005). Dans le cadre de la présente étude, les prospections effectuées en zones sahélienne et sahélo-soudanienne ont permis de recenser seulement 28 espèces ligneuses avec une dominance des Mimosaceae dont le genre le plus représenté est Acacia avec 5 espèces.

En dépit de ces conditions écologiques très difficiles, certaines plantes ont développé des stratégies leur permettant de s'adapter et de perpétuer leurs espèces (Mahamane et Saadou, 2009). Parmi les stratégies utilisées on peut citer le développement d'épines et la formation des faisceaux libéro-ligneux surnuméraires leur permettant d'emmagasiner une grande quantité d'eau qu'elles utilisent en saisons sèches très longues caractéristiques des zones arides. L'adoption de ces stratégies a été signalée chez B. aegyptiaca et Z. mauritiana (Mahamane et Saadou, 2009). Plusieurs études ont montré que ces deux espèces sont en pleine expansion en zone sahélienne alors que dans le cadre de la présente étude des structures perturbées ont été observées. L'analyse de l'indice de valeur d'importance a montré que $B$. aegyptiaca et $Z$. mauritiana sont les espèces les plus importantes dans l'ensemble des sites prospectés. Cela s'explique par le fait que la plupart des relevés ont été effectués dans les zones où ces deux espèces sont présentes. En effet, dans ces zones, la forte densité et la vigueur des individus de ces deux espèces leur confèrent une dominance dans une végétation dominée par des microphanérophytes. Des résultats similaires ont été observés dans la Commune de Libori au Niger par Idrissa et al. (20I8) avec des valeurs d'indice d'importance plus élevées pour B. aegyptiaca (170\%). L'analyse de la densité des peuplements a permis de constater que le site de Kegil est plus dense en plantes ligneuses avec 333,I士IOI,7 arbre/ha. Cette forte densité s'explique par l'hétérogénéité des sites ayant fait l'objet des relevés. En effet, dans ce dernier site la plupart des relevés ont été effectués dans la forêt classée de Madarounfa où subsistent encore les peuplements de Z . mauritiana et $\mathrm{B}$. aegyptiaca. Les différences de densités des adultes observées entre les secteurs s'expliquent par la différence de type d'occupation des terres. En effet, les observations ont été effectuées en grande partie dans les formations naturelles dans le secteur sahélo-soudanien, alors que dans le secteur sahélien strict ce sont des champs qui ont fait l'objet de prospections. Les caractéristiques morphologiques notamment, la présence des épines chez ces deux espèces, les rendent indésirables dans les champs surtout en zone sahélo-soudanienne où la ressource ligneuse est encore disponible du fait des conditions pluviométriques plus favorables. Selon les cultivateurs, les épines de ces espèces entravent les activités champêtres et par conséquent, elles sont défrichées au début de chaque saison des pluies. Par contre la faible densité observée en zone sahélienne s'explique par le fait que l'échantillonnage concerne plus les champs. Dans ce dernier secteur, les conditions pluviométriques non favorables obligent les populations à conserver dans les champs même les espèces indésirables. Les travaux effectués par Baggnian et al. (2014) sur la régénération naturelle assistée à Dan Saga 
corroborent les résultats du présent travail. En effet, ces travaux ont montré la forte implication des paysans dans l'enrichissement et la conservation des espèces ligneuses dans les champs.

Les plus gros diamètres moyens de Z. mauritiana observés sur le site de Birni Lallé, en dépit de son appartenance au secteur sahélien strict s'explique par le fait que les peuplements ayant fait l'objet d'inventaire ne sont présents que dans une dépression autour d'une mare où les conditions sont favorables au développement de la végétation. Plus encore, sur ce dernier site on constate que le taux de régénération et la densité des juvéniles sont plus élevés. Khamis et al. (2015) ont montré que l'arbre de Z. mauritiana est résistant à la sécheresse et au feu et supporte jusqu'à 2 mois d'inondation dans les zones près de la rivière.

En secteur sahélo-soudanien, les fortes densités de B. aegyptiaca et de Z. mauritiana observées sur le site de Kegil s'explique par les conditions stationnaires. Dans cette dernière zone, nos relevés ont été effectués dans un parc à $B$. agyptiaca et $Z$. mauritiana. Dans ce parc, les deux espèces constituent des peuplements purs avec une densité pouvant aller jusqu'à 390 arbres de B. aegyptica par hectare. Ce dernier site est suivi du site de Birni Lallé dans le secteur sahélien où ces deux espèces peuvent être observées même dans les champs. Le site de Dan Saga, le site le moins arrosé de nos zones d'étude, constitue le deuxième site où la densité de $\mathrm{B}$. aegyptiaca est importante après le site de Kegil. En effet, le village de Dan Saga constitue une référence au Niger en matière de régénération naturelle assistée (RNA) (Baggnian et al., 20I4).

La différence entre les types d'occupations des terres et les conditions climatiques au niveau des stations prospectées influencent la distribution des paramètres structuraux des peuplements ligneux dans la zone de la présente étude. L'analyse des paramètres structuraux sur sol de textures différentes et de type d'occupation des sols a permis de mettre en évidence les meilleurs préférendums écologiques de B. aegyptiaca et Z. mauritiana. Le sol argileux observé au niveau des dépressions de par ses caractéristiques hydromorphes offre de meilleures conditions favorables à leurs croissances. Cela explique les diamètres et hauteurs moyens de B. aegyptiaca significativement élevés. Un autre facteur influençant les paramètres structuraux est le statut des sites qui hébergent les peuplements. En effet, des différences significatives des paramètres structuraux ont été observées entre les champs, les aires de pâturage et les forêts classées aussi bien sur B. aegyptiaca que sur Z. mauritiana. Plusieurs auteurs ont signalé l'influence de la conservation sur les paramètres structuraux des arbres (Rabiou et al., 2015; Savadogo et al., 20ı6)

La fréquence des plantules issues de drageonnage est toujours supérieure à celle des plantules issues de la germination. Ces différences sont observées dans tous les secteurs et au niveau de toutes les deux espèces. Les plantules issues de la germination ont moins de chance de survivre dans les champs surtout en zone sahélo-soudanienne où elles sont indésirables. Par contre les drageons même défrichés, végètent encore à la fin des saisons des pluies après l'abandon des champs. Ces aspects montrent l'importance du mode d'adaptation et de la recolonisation des zones arides par ces deux espèces. Les travaux effectués en zone sahélienne par Bellefontaine et al. (2005) ont montré que Guiera 
senegalensis et Combrteum micranthum, deux espèces dominantes dans les brousses tigrées, utilisent ces stratégies pour constituer l'essentiel de la végétation. La distance moyenne entre le drageon et l'arbre mère est plus importante au niveau de B. aegytiaca et plus faible au niveau de Z. mauritiana même si par ailleurs des différences significatives n'ont pas été observées entre les secteurs.

L'analyse des classes de diamètre a permis d'appréhender la dynamique des peuplements de B. aegyptiaca. Les peuplements de l'espèce ont présenté la meilleure structure dans le secteur sahélien. Les individus jeunes sont relativement présents dans les deux villages. Dans ce secteur, ces deux espèces en dépit de leurs caractéristiques épineuses, sont conservées dans les champs du fait de la rareté de la ressource dans la zone. Dans l'ensemble des relevés effectués à Birni Lallé, 5 espèces seulement ont été recensées. La conservation de B. aegyptiaca et Z. mauritiana s'impose puisqu'elles constituent un maillon d'une faible chaîne des ressources de la zone. A Dan Saga B. aegyptiaca est non seulement conservé dans le cadre de la régénération naturelle assistée mais aussi pour son importance socio-économique dans le village. En effet, dans ce village, les femmes ont développé une unité de production d'huile et de savon en transformant les fruits de B. aegyptiaca. Pendant la période de fructification de B. aegyptiaca, une des principales activités des femmes est la collecte des fruits de cette espèce. Cette même activité est également pratiquée par les femmes dans les deux villages du secteur sahélo-soudanien notamment à Kegil et à Mai Guéro. En dépit de l'importance de cette activité génératrice de revenus pour les femmes, l'espèce n'est pas conservée dans les champs au niveau de ce dernier secteur. Cela constitue une source des conflits entre les femmes qui tirent des revenus de l'exploitation de ces espèces et les hommes propriétaires des champs qui sont gênés par les épines au cours des travaux champêtres. Des observations similaires ont été effectuées par Bationo et al. (2005) au Burkina Faso.

Les deux espèces ne sont présentes que dans les aires de pâturage et dans les forêts classées. Ces deux unités d'occupations sont constamment parcourues par les troupeaux d'animaux avec comme conséquences, le piétinement et le broutage des jeunes plantes, réduisant ainsi la capacité de résilience de ces écosystèmes. Les obstacles occasionnés à la régénération par le pâturage dans ces unités se traduit par une distribution en cloche des classes de diamètres observées au niveau des deux sites du secteur sahélo-soudanien. Les mêmes observations sont valables pour Z. mauritiana. En effet, le ramassage des fruits de l'espèce appauvrit la banque séminale édaphique et compromet la régénération de l'espèce. Les individus jeunes et les individus adultes sont faiblement représentés. Les individus de classes moyennes sont relativement les plus abondants. Plusieurs auteurs ont montré la relation qui existe entre les jeunes et les sujets adultes (Thiombiano et al., 2006; Glélé et al., 2008; Assogbadjo et al., 2009; Adjonou et al., 2010; Bationo et al., 2010). En effet, les plantules assurent l'avenir du peuplement et les sujets adultes assurent la production des semences pour le renouvellement du peuplement (Dan Guimboet al., 20I0; Adjonou et al., 2010). 


\section{Conclusion}

l'importance socio-économique de B. aegyptiaca et $\mathrm{Z}$. mauriatina a été la principale motivation du choix de ces deux espèces pour leur analyse démographique. L'analyse de leurs peuplements dans les formations naturelles et dans les champs a permis de dégager des conclusions sur leur état actuel. En dépit de l'exploitation de leurs fruits, les deux espèces restent encore disponibles dans toutes les zones agro-écologiques de la région de Maradi. Dans le secteur sahélien, compte tenu de la rareté des ressources végétales, la densité de ces espèces est importante dans les champs et dans les formations naturelles. Par contre dans le secteur sahélo-soudanien où la ressource est abondante, la densité élevée de ces deux espèces est observée essentiellement dans les formations naturelles. Elle est faible au niveau des champs du fait de la présence des épines gênant ainsi les paysans lors des travaux champêtres. L'étude a également montré la capacité d'adaptation de ces espèces en zones arides à travers les différentes formes de multiplication végétative adoptées par ces espèces. Ces aptitudes de propagation végétatives expliquent la prédominance et à la perpétuation de ces espèces en zone aride. La conservation de ces espèces peut continuer à garantir la résilience de leurs populations dans les environnements arides affectés par le changement climatique et d'affectation des terres.

\section{Remerciement}

À AGROPILIS à travers le financement du Projet 'Amélioration de la disponibilité d'aliments nutritifs par la promotion d'espèces d'arbres/arbustes comestibles indigènes en Afrique subsaharienne"' (TREEFOOD).

\section{Références bibliographiques}

Abdou, M. M. (2014). États de surface et fonctionnement hydrodynamique multi-échelles des bassins sahéliens; études expérimentales en zones cristalline et sédimentaire. Sciences de la Terre. Université de Grenoble, Français, 268 p.

Adjonou, K., Ali, N., Kokutse, A. D. \& Kokou, K. (20I0). Étude de la dynamique des peuplements naturels de P. erinaceus Poir. (Fabaceae) surexploités au Togo. Bois et Forêts des Tropiques, 306 (I), pp $33-43$.

Adomou, C.A., Mama, A., Missikpode, R. \& Sinsin, B. (2009). Cartographie et caractérisation floristique de la forêt marécageuse de Lokoli (Bénin). International Journal of Biological and Chemestry Sciences, 3(3): 492-503.

Ambouta, K. (1984). Contribution à l'édaphologie de la brousse tigrée de l'ouest nigérien. Thèse de Doctorat Ingénieur, Université de Nancy I, France, Ir6p.

Assogbadjo, A. E., Glélé Kakaï, R., Sinsin, B. \& Pelz, D. (2009). Structure of Anogeissus leiocarpa Guill., Perr. natural stands in relation to anthropogenic pressure within Wari-Maro Forest Reserve in Benin. African Journal of Ecology, I-IO. doi: IO.IIII/j.I365-2028.2009.0II6o.x

Baggnian, I., Adam, T., Adamou, M.M., Chaibou, I. \& Mahamane, A. (20I4). Structure etdynamique de la végétation ligneusejuvénile issue de la régénération naturelle assistée (RNA) dans le Centre-Sud du Niger. International Journal of Biological and Chemestry Sciences, 8(2): 649-665.

Bationo, B. A., Alkassoum, Maïga A., Compaoré, P. \& Antoine Kalinganire, A. (2010). Dimension socioculturelle du baobab (Adansonia digitata L.) dans le Plateau central du Burkina Faso. Bois et Forêts des Tropiques, 306 : 23-32. 
Bellefontaine, R., Sabir, M., Kokou, K., Guinko, S., Saadou, M., Ichaou, A., Hatem, C., Bationo, B.A., Karim, S. \& Dourma, M. (2005). Argumentaire pour l'étude et l'utilisation des marcottes et drageons dans les pays à faible couvert ligneux. Sècheresse, I6(4): 312-3I4.

Bationo, B.A., Ouedraogo, S.J., Some, N.A., Pallo, F. \& Boussim, I. J. (2005). Ecologie de la régénération naturelle de Isoberlinia doka Craib. et stapf. dans la forêt classée de Nazinon (Burkina Faso). Cahiers d'Agriculture, I4(3) : I-9.

Chothani, D.L. \& Vaghasiya, H.U. (20II). A review on Balanites aegyptiaca Del (desert date): phytochemical constituents, traditional uses, and pharmacological activity. Pharmacognosy Review, 5(9):55-62.DOI:10.4103/0973-7847.79100.

Dan Guimbo, I., Mahamane, A. \& Ambouta, K. J. M. (2010). Peuplement des parcs à Neocarya macrophylla (Sabine) Prance et à Vitellaria paradoxa (Gaertn. C.F.) dans le sud-ouest nigérien : diversité, structure et régénération. International journal of biological and chemestry science, 4(5): 1706I720.

Deblauwe, V. (2010). Modulation des structures de végétation auto-organisées en milieu aride. These de doctorat, ULB. I73p.

Doughari, J.H., Pukuma, M.S., De, N. (2007). Antimalarial effects of Balanites aegyptiaca L. Drel. and Moringa oleifera Lam. on Salmonella typhi. African Journal of Biotechnology 6, 2212-2215.

FAO. (2009). Situation des forêts du monde. Rome, Italie. I68 p. ISBN 978-92-5-206057-4.

FAO. (2010). Évaluation des ressources forestières mondiales 20I0: Rapport principal. Étude FAO: Forêts r63p. Rome. www.fao.org/docrep/or3/ir757f/ir757f.pdf.

FAO. (2012). Situation des forêts du monde. 66 p., ISBN 978-92-5-207292-8.

Glèlè, K.R., Sinsin, B. \& Palm, R. (2008). Étude dendrométrique de P. erinaceus Poir. des formations naturelles de la zone soudanienne au Bénin. Agronomie africaine, 20 (3) : 245-255.

Hassane, H. (2008). Répertoire des espèces végétales les plus couramment utilisées en pharmacopée traditionnelle et impact des techniques de prélèvement sur la diversité biologique dans la réserve de Biosphère $d u$ $W$ du Niger. Mémoire de DEA, Université Abdou Moumouni. II3 p.

Ibrahim, H., Abdurrahman, E.M., Shok, M., Ilyas, N., Musa, K.Y. \& Ikandu, I. (2007).Comparative analgesic activity of the root bark, stem bark, leaves, fruits and seed of Carissa edulis VAHL (Apocynaceae). African Journal of Biotechnology, 6:1233-1235.

Idrissa, B., Soumana, I., Issiaka, Y., Ambouta, J.M.K., Mahamane, A., Saadou, M. \& Weber J. C. (20I8). Trend and Structure of Populations of Balanites agyptiaca in Parkland Agroforests in Western Niger. Annual Research \& Review in Biology, 22(4): I-I2.

Jamilu, Y., Yaro, A.H., Magaji, M.G., Abubakar, M.S., Sani, M., Maje, I.M., Hussaini, I.M. \& Anuka, J.A. (2008). Anticonvulsant activity of hydro-alcoholic root bark extract ofBalanitesaegyptiaca (L.) Del. Biological and Environmental Sciences Journal for the Tropics 5: 5-9.

Khamis, G., Schaar Schmidt, F. \& Papenbrock, J. (2015). Effect of water deficiency on different genotypes of Balanites aegyptiaca. Procedia Environmental Sciences, 29: 49 - 50.

Koko, W.S., Galal, M. \& Khalid, H.S. (2000). Fasciolicidal efficacy of Albizia antihelmintica and Balanites aegyptiaca compared with albendazole. Journal of Ethnopharmacology, 7I : 247-252.

Louppe, D., Herault, B., Zo-Bi, I.C. \& Kouadio, K.B. (2018). Révision des normes d'aménagement des forêts classées de Côte d'Ivoire. Rapport d'expertise scientifique, Cirad, 3op + annexes.

Mahamane, A., Saadou, M., Bakasso, Y., Abassa, I., Aboubacar, I. \& Karim, S. (2007). Analyse diachronique de l'occupation des terres et caractéristiques de la végétation dans la commune de Gabi (Région de Maradi, Niger). Sécheresse, I8(4) : 296-304.

Mahamane, A. \& Saadou, M. (2009). Structures anatomiques de quelques organes de Boscia senegalensis (Pers.) Lam. ex Poir. Et adaptation a' la sécheresse. Sécheresse, 20 (2) : 237-9.

Maruza, I.M., Musemwa, L., Mapurazi, S., Matsika, P., Munyati, V.T. \& Ndhleve, S. (2017). Future prospects of Ziziphus mauritiana in alleviating household food insecurity and illnesses in arid and semi-arid areas: A review. World Development Perspectives, 5: I-6. 
Mounkaila, S., Barmo, S., Morou, B., Saley, K., Bil-Assanou, H.I., Mahamane, A., Ikhiri, K. \& Saadou, M. (2017). Inventaire Et Gestion Des Plantes Médicinales Dans Quatre Localités Du Niger. European Scientific Journal, 24(13) : 498-521.

Ojo, O.O., Nadro, M.S. \& Tella, I.O. (2006). Protection of rats by extracts of some commonNigerian trees against acetaminophen-induced hepatotoxicity. African Journal of Biotechnology, $5: 755-760$.

Ozer, A. \& Ozer, P. (2005). Désertification au Sahel: crise climatique ou anthropique? Bulletin des Séances de l'Académie royale des sciences d'Outre-Mer, $5 \mathrm{I}: 395-423$.

Rabiou, H., Diouf, A., Bationo, B. A., Segla, K.N., Adjonou, K., Kokutse, A.D., Radji, R., Kokou, K., Mahamane, A. \& Saadou, M. (2015). Structure des peuplements naturels de Pterocarpus erinaceusPoir. dans le domaine soudanien, au Niger et au Burkina Faso. Bois et forêts des tropiques, $325(3): 71-83$.

Rondeux, J. (1999). La mesure des arbres et des peuplements forestiers. Gembloux, Belgique, Les Presses agronomiques de Gembloux, 2e édition, 52I p.

Saotoing, P., Vroumsia, T., Tchobsala, Tchuenguem, F.F-N., Njan Nloga, A.-M. \& Messi, J. (20II). Medicinal plants used in traditional treatment of malaria in Cameroon. Journal of Ecology and the Natural Environment, 3(3) : I04-II7.

Savadogo, O.M., Ouattara, K., Pare, S., Ouedraogo, I., Sawadogo-Kaboré, S., Barron, J. \& Zombre P.N. (20I6). Structure, composition spécifique et diversité des ligneux dans deux zones contrastées en zone Sahélienne du Burkina Faso. Vertigo, r6 (I) : 2-16.

Sinsin, B., Eyog Matig, O., Assogbadjo, A.E., Gaoué, O.G. \& Sinadouwirou, T. (2004). Dendrometric characteristics as indicators of pressure of Afzelia africana Sm. Dynamic changes in trees found in different climatic zones of Benin. Biodiversity and conservation, I3: 1555-I570.

Thiombiano, A., Schmidt, M., Kreft, H. \& Guinko, S. (2006). Influence du gradient climatique sur la distribution des espèces de Combretaceae au Burkina Faso (Afrique de l'ouest). Jour. Inter. Botanique Système, Candollea, 6I(I) : 189-213.

Vantomme, P. \& Gazza, S. (2010). Le défi de la sylviculture en faveur des produits forestiers non ligneux sous les tropiques :de la cueillette à l'agriculture? Bois et forêts des tropiques, 304 ( 2): 5-13.

Wang, B., Zhu, T., Wang, D., Yang, C-R., Xu, M., \& Zhang, Y-J. (2013). New spinosin derivatives from the seeds of Ziziphus mauritiana. Natural Produce Bioprospective, 3:93-98. DOI ro.1007/si3659-013$0028-5$.

White, F. (1983). La végétation de l'Afrique. Mémoire accompagnant la carte de végétation de l'Afrique. L'Organisation des Nations Unies pour l'Éducation, la Science et la Culture (UNESCO).

Yadav, J.P. \& Panghal, M. (2010). Balanites aegyptiaca (L.) Del. (Hingot): a review of its traditional uses, phytochemistry and pharmacological properties. International Journal of Green Pharmacy, 4: I40-I46. 